\title{
The Existence of a Global Attractor for one Fourth Order Hyperbolic Equation with Memory Operator
}

\author{
Sevda Elkhan Isayeva ${ }^{1}$ \\ ${ }^{1}$ Baku State University, 23 Academician Z.Khalilov Str., AZ1148, Baku, Azerbaijan
}

\section{Article Info}

Keywords: Bounded absorbing set, Memory operator, Minimal global attractor, Semilinear hyperbolic equation 2010 AMS: 35L30, $35 L 76$

Received: 26 April 2018

Accepted: 29 November 2018

Available online: 20 March 2019

\begin{abstract}
In this work, the initial-boundary value problem for one fourth order semilinear hyperbolic equation with memory operator is considered (here the memory operator is under the operator of differentiation with respect to time variable). The asymptotic compactness of semigroup generated by this problem is proved. The existence of a minimal global attractor for this problem is also proved.
\end{abstract}

\section{Introduction}

Nonlinear equations with memory operator, especially the equations with hysteresis have great importance among the partial differential equations. Nonlinear relations of hysteresis type appear in ferromagnetism, ferroelectricity, superconductivity, plasticity, friction, etc. The research of solutions of partial differential equations with hysteresis nonlinearities is a nontrivial problem. Such equations, when hysteresis operator is under the operator of differentiation with respect to time variable, have special difficulties.

The research of asymptotic behaviour of a dynamic system, which is originated by the corresponding initial-boundary value problem, has a special significance. For the equations with hysteresis nonlinearities, these questions have not been almost investigated. In this field, only particular cases were considered. For instance, the asymptotic character of solutions of the initial-boundary value problem for one quasilinear parabolic equation, in which the hysteresis operator is under the operator of differentiation with respect to time variable, was investigated in [1]. The similar results were obtained in [2], [3]. In [4], [5], [6] the corresponding problems were researched by the application of the results of nonlinear semigroup theory.

In this work, the asymptotic result for solutions of the initial-boundary value problem for one semilinear hyperbolic equation with memory operator is obtained and the existence of a minimal global attractor for this problem is proved.

\section{Problem statement and reliminaries}

Here we use the concepts and notations which were introduced in [7].

Let $\Omega \subset R^{N}(N \geq 1)$ be a bounded, connected set with a smooth boundary $\Gamma$. We consider the following problem:

$$
\begin{aligned}
& \frac{\partial^{2} u}{\partial t^{2}}+\frac{\partial}{\partial t}[u+\mathrm{F}(u)]+\Delta^{2} u+|u|^{p} u=h \text { in } Q=\Omega \times(0, T), \\
& u=0, \Delta u=0,(x, t) \in \Gamma \times[0, T], \\
& {\left.[u+\mathrm{F}(u)]\right|_{t=0}=u^{(0)}+w^{(0)},\left.\frac{\partial u}{\partial t}\right|_{t=0}=u^{(1)} \operatorname{in} \Omega,}
\end{aligned}
$$


where $p>0$ and $\mathrm{F}$ is a memory operator (at any instant t, $\mathrm{F}(u)$ may depend not only on $u(t)$, but also on the previous evolution of $u$ ), which acts from $\mathrm{M}\left(\Omega ; C^{0}([0, T])\right)$ to $\mathrm{M}\left(\Omega ; C^{0}([0, T])\right)$. Here $\mathrm{M}\left(\Omega ; C^{0}([0, T])\right)$ is a space of strongly measurable functions $\Omega \rightarrow C^{0}([0, T])$. We assume that the operator $\mathrm{F}$ is applied at each point $x \in \Omega$ independently: the output $[\mathrm{F}(u(x, \cdot))](t)$ depends on $\left.u(x, \cdot)\right|_{[0, t]}$, but not on $\left.u(y, \cdot)\right|_{[0, t]}$ for any $y \neq x$ (for more details see [7]).

We assume that

$$
\left\{\begin{array}{l}
\text { for } \forall v_{1}, v_{2} \in \mathrm{M}\left(\Omega ; \mathrm{C}^{0}([0, \mathrm{~T}])\right) \text { and for } \forall \mathrm{t} \in[0, \mathrm{~T}], \text { if } v_{1}=v_{2} \\
\text { in }[0, \mathrm{t}], \text { a.e. in } \Omega, \text { then }\left[\mathrm{F}\left(v_{1}\right)\right](\cdot, \mathrm{t})=\left[\mathrm{F}\left(v_{2}\right)\right](\cdot, \mathrm{t}) \text { a.e. in } \Omega ;
\end{array}\right.
$$

$$
\left\{\begin{array}{l}
\forall\left\{v_{n} \in \mathrm{M}\left(\Omega ; C^{0}([0, T])\right)\right\}_{n \in \mathrm{N}}, \text { if } v_{\mathrm{n}} \rightarrow v \text { uniformly in }[0, \mathrm{~T}] \\
\text { a.e.in } \Omega, \text { then } \mathrm{F}\left(v_{\mathrm{n}}\right) \rightarrow \mathrm{F}(v) \text { uniformly in }[0, \mathrm{~T}] \text { a.e.in } \Omega ;
\end{array}\right.
$$

$$
\left\{\begin{array}{l}
\exists L>0, \exists g \in L^{2}(\Omega): \forall v \in M\left(\Omega ; C^{0}([0, T])\right) \\
\|[\mathrm{F}(v)](x, \cdot)\|_{C^{0}([0, T])} \leq L\|v(x, \cdot)\|_{C^{0}([0, T])}+g(x), \text { a.e. in } \Omega ;
\end{array}\right.
$$

$$
\left\{\begin{array}{l}
\forall v \in \mathrm{M}\left(\Omega ; C^{0}([0, T])\right), \forall\left[t_{1}, t_{2}\right] \subset[0, T], \\
\text { if } v(\mathrm{x}, \cdot) \text { is affine in }\left[\mathrm{t}_{1}, \mathrm{t}_{2}\right] \text { a.e. in } \Omega, \text { then } \\
\left\{[\mathrm{F}(v)]\left(x, t_{2}\right)-[\mathrm{F}(v)]\left(x, t_{1}\right)\right\}\left[v\left(x, t_{2}\right)-v\left(x, t_{1}\right)\right] \geq 0, \text { a.e. in } \Omega ;
\end{array}\right.
$$

$$
\left\{\begin{array}{l}
\exists 0<L_{1}<1, \forall v \in M\left(\Omega ; C^{0}([0, T])\right), \forall\left[t_{1}, t_{2}\right] \subset[0, T], \\
\text { if } v(x, \cdot) \text { is affine in }\left[t_{1}, t_{2}\right] \text { a.e.in } \Omega, \text { then } \\
\left|[\mathrm{F}(v)]\left(x, t_{2}\right)-[\mathrm{F}(v)]\left(x, t_{1}\right)\right| \leq L_{1}\left|v\left(x, t_{2}\right)-v\left(x, t_{1}\right)\right| \text { a.e.in } \Omega .
\end{array}\right.
$$

$$
\left\{\begin{array}{l}
\exists 0<L_{2}<1, \forall v \in M\left(\Omega ; C^{0}([0, T])\right), \forall\left[t_{1}, t_{2}\right] \subset[0, T], \\
\text { if } v(x, \cdot) \text { is affine in }\left[t_{1}, t_{2}\right] \text { a.e.in } \Omega, \text { then } \\
\left|[\mathrm{F}(u)]\left(x, t_{2}\right)-[\mathrm{F}(v)]\left(x, t_{2}\right)-\left([\mathrm{F}(u)]\left(x, t_{1}\right)-[\mathrm{F}(v)]\left(x, t_{1}\right)\right)\right| \leq \\
\leq L_{2}\left|u\left(x, t_{2}\right)-v\left(x, t_{2}\right)-\left(u\left(x, t_{1}\right)-v\left(x, t_{1}\right)\right)\right| .
\end{array}\right.
$$

Let $V=H_{0}^{2}(\Omega) \cap L^{p+2}(\Omega)$ and

$$
u^{(0)} \in V, w^{(0)} \in L^{2}(\Omega), u^{(1)} \in L^{2}(\Omega), h \in L^{2}(\Omega) .
$$

Definition 2.1. A function $u \in L^{2}(0, T ; V) \cap H^{1}\left(0, T ; L^{2}(\Omega)\right)$ is said to be a solution of problem (2.1)-(2.3) if $\mathrm{F}(u) \in L^{2}(Q)$, and

$$
\begin{aligned}
& \iint_{Q}\left\{-\frac{\partial u}{\partial t} \cdot \frac{\partial v}{\partial t}-[u+\mathrm{F}(u)] \frac{\partial v}{\partial t}+\Delta u \cdot \Delta v+|u|^{p} u v\right\} d x d t= \\
& =\iint_{Q} h v d x d t+\int_{\Omega}\left[u^{(0)}(x)+w^{(0)}(x)+u^{(1)}(x)\right] v(x, 0) d x
\end{aligned}
$$

for every $v \in L^{2}(0, T ; V) \cap H^{1}\left(0, T ; L^{2}(\Omega)\right)(v(\cdot, T)=0$ a.e. in $\Omega)$.

Well posedness of problem (2.1)-(2.3) without F, was studied by different authors (see, for example [8]). The initial boundary problem for the parabolic equation without the nonlinear term $|u|^{p} u$ and with $\Delta u$ was studied in [7].

The following theorems about existence and uniqueness of solutions of problem (2.1)-(2.3) can be proved in the same way as the corresponding theorems from [9].

Theorem 2.2. Assume that (2.4)-(2.8),(2.10) hold. Then problem (2.1)-(2.3) has at least one solution such that

$$
u \in W^{1, \infty}\left(0, T ; L^{2}(\Omega)\right) \cap L^{\infty}(0, T ; V), \mathrm{F}(u) \in H^{1}\left(0, T ; L^{2}(\Omega)\right) .
$$

Theorem 2.3. Assume that the hypotheses of Theorem 2.2 hold,

$$
p \leq \frac{2}{N-2}, N \geq 3(p \text { is arbitrary and finite when } N=2)
$$

and $F$ fulfils the following condition

$$
\left\{\begin{array}{l}
\forall r>0, \exists L(r)>0: \forall t \in(0, T], \forall v_{1}, v_{2} \in\left\{u \in L^{2}\left(Q_{t}\right):\|u\|_{L^{2}\left(Q_{t}\right)} \leq r\right\}: \\
\left\|F\left(v_{1}\right)-F\left(v_{2}\right)\right\|_{L^{2}\left(Q_{t}\right)} \leq L(r)\left\|v_{1}-v_{2}\right\|_{L^{2}\left(Q_{t}\right)} .
\end{array}\right.
$$

Then problem (2.1)-(2.3) has only one solution. 
As an example of an operator which satisfies the mentioned conditions, we can present the Bouc operator (see, for example [10]):

$$
[B(u)](t)=\alpha u(t)+\int_{0}^{t} f\left(\int_{s}^{t}\left|u^{\prime}(\tau)\right| d \tau\right) \varphi(u(s)) u^{\prime}(s) d s,
$$

here $\alpha$ is a positive constant, $f$ and $\varphi$ are continuous real functions, with $f$ positive and nondecreasing.

The following theorem is obtained from Theorem 2.2 and Theorem 2.3 by the common theory of solvability of linear hyperbolic equations.

Theorem 2.4. Assume that the conditions of Theorem 2.3 hold. Then for arbitrary $T>0$ problem (2.1)-(2.3) has only one solution $u \in C^{1}\left([0, T] ; L^{2}(\Omega)\right) \cap C\left([0, T] ; H_{0}^{2}(\Omega)\right)$.

By the condition (2.11): $V=H_{0}^{2}(\Omega) \cap L^{p+2}(\Omega)=H_{0}^{2}(\Omega)$. We set $E=H_{0}^{2}(\Omega) \times L^{2}(\Omega) \times L^{2}(\Omega)$. Then under the conditions of Theorem 2.4, Problems (2.1)-(2.3) generates the semigroup $\{S(t)\}_{t \geq 0}$ in $E$ by the formula:

$$
S(t)\left(u^{(0)}, u^{(1)}, w^{(0)}\right)=\left(u, u_{t}, w\right)
$$

where $u$ is a unique solution of this problem.

Definition 2.5. (see [11]) $A$ bounded set $B_{0} \subset E$ is said to be absorbing iffor an arbitrary bounded set $B \subset E$ there exists $t_{1}(B)$ such that $S(t) B \subset B_{0}$ for all $t \geq t_{1}(B)$.

Definition 2.6. (see [11]) Let $\{S(t)\}_{t \geq 0}$ be a semigroup on a metric space $(X, d)$. A smallest, nonempty, bounded, closed set $A \subset X$ that satisfies

$$
\lim _{t \rightarrow \infty} \sup _{v \in B} \inf _{u \in A} d(S(t) v, u)=0,
$$

for each bounded set $B \subset X$, is called a minimal global attractor of $\{S(t)\}_{t \geq 0}$.

The following theorem about the existence of a bounded absorbing set for Problems (2.1)-(2.3) can be proved in the same way as the corresponding theorem in [12].

Theorem 2.7. Under the conditions (2.4)-(2.12), Problems (2.1)-(2.3) has a bounded absorbing set $B_{0} \subset E$.

In this work, we first prove the asymptotic compactness of a semigroup, generated by problem (2.1)-(2.3), and then the basic theorem about the existence of a minimal global attractor for this problem.

Note that, a semigroup $\{S(t)\}_{t \geq 0}$, defined on a metric space $(X, d)$, is called asymptotically compact, if for arbitrary bounded set $B \subset X$ such, that $\bigcup_{t \geq 0} S(t) B$ is bounded in $(X, d)$, the sequence $\left\{S\left(t_{k}\right) v_{k}\right\}_{k=1}^{\infty}, t_{k} \rightarrow \infty, v_{k} \in B$ has a convergent subsequence.

\section{Basic Results}

Theorem 3.1. Assume that (2.4)-(2.12) hold. Then the semigroup $\{S(t)\}_{t \geq 0}$, generated by problem (2.1)-(2.3), is asymptotically compact in $E$.

The proof of Theorem 3.1. It suffices to prove that for any bounded set $B$ from $E$ and for arbitrary $\varepsilon>0$ there exists $T=T(\varepsilon, B)$ such that

$$
\limsup _{i \rightarrow \infty} \sup _{q \in N}\left\|S(T) \theta_{i+q}-S(T) \theta_{i}\right\|_{E} \leq \varepsilon,
$$

where $\left\{\theta_{i}\right\}$ is the sequence from $B$ and $\left\{S(t) \theta_{i}\right\}$ converges $*$-weekly in $L^{\infty}(0, \infty ; E)$.

We prove this by the method of time discretization (see [7]).

For any $m \in N$, we set $k=\frac{T}{m}$ and:

$$
\begin{aligned}
& u_{m}^{0}=u^{(0)}, w_{m}^{0}=w^{(0)}, u_{m}^{1}=u^{(0)}+k u^{(1)}, \\
& u_{m}^{-1}=u^{(0)}-k u^{(1)}, u_{m}^{n}(x)=u(x, n k), n=2, \ldots, m, \\
& w_{m}^{n}(x)=\left[\mathrm{F}\left(u_{m}\right)\right](x, n k), n=1, \ldots, m, \text { a.e. in } \Omega,
\end{aligned}
$$

$u_{m}(x, \cdot)=$ linear time interpolate of $u(x, n k)$ for $n=0,1, \ldots, m$ a.e. in $\Omega, w_{m}(x, \cdot)=$ linear time interpolate of $w(x, n k)$ for $n=1, \ldots, m$ a.e. in $\Omega$. We will use the techniques used in $[13,14,15]$. We set $\theta_{i}=\left\{u_{i}^{(0)}, u_{i}^{(1)}, w_{i}^{(0)}\right\}, u_{i}=S(t) \theta_{i}(i=1,2, \ldots)$.

We consider the following problems for $l=i, j(i, j=1,2, \ldots)$ :

$$
\begin{gathered}
\frac{u_{l m}^{n}-2 u_{l m}^{n-1}+u_{l m}^{n-2}}{k^{2}}+\frac{u_{l m}^{n}-u_{l m}^{n-1}}{k}+\frac{w_{l m}^{n}-w_{l m}^{n-1}}{k}+ \\
+\Delta^{2} u_{l m}^{n}+\left|u_{l m}^{n}\right|^{p} u_{l m}^{n}=h \text { in } V^{\prime}, n=1,2, \ldots, m, \\
u_{l m}^{0}=u_{l}^{(0)}, w_{l m}^{0}=w_{l}^{(0)}, u_{l m}^{1}=u_{l}^{(0)}+k u_{l}^{(1)}, u_{l m}^{-1}=u_{l}^{(0)}-k u_{l}^{(1)},
\end{gathered}
$$

and obtain, that

$$
\frac{u_{i m}^{n}-u_{j m}^{n}-2\left(u_{i m}^{n-1}-u_{j m}^{n-1}\right)+u_{i m}^{n-2}-u_{j m}^{n-2}}{k^{2}}+\frac{u_{i m}^{n}-u_{j m}^{n}-\left(u_{i m}^{n-1}-u_{j m}^{n-1}\right)}{k}+
$$




$$
\begin{aligned}
& +\frac{w_{i m}^{n}-w_{j m}^{n}-\left(w_{i m}^{n-1}-w_{j m}^{n-1}\right)}{k}+ \\
& +\Delta^{2}\left(u_{i m}^{n}-u_{j m}^{n}\right)+\left|u_{i m}^{n}\right|^{p} u_{i m}^{n}-\left|u_{j m}^{n}\right|^{p} u_{j m}^{n}=0, \text { in } V^{\prime}, n=1,2, \ldots, m . \\
& u_{l m}^{0}-u_{j m}^{0}=u_{l}^{(0)}-u_{j}^{(0)}, w_{l m}^{0}-w_{j m}^{0}=w_{l}^{(0)}-w_{j}^{(0)}, \\
& u_{l m}^{1}-u_{j m}^{1}=u_{l}^{(0)}-u_{l}^{(0)}+k\left(u_{l}^{(1)}-u_{j}^{(1)}\right), u_{l m}^{-1}-u_{j m}^{-1}=u_{l}^{(0)}-u_{l}^{(0)}-k\left(u_{l}^{(1)}-u_{j}^{(1)}\right) .
\end{aligned}
$$

By multiplying both sides of equality (3.2) by $u_{i m}^{n}-u_{j m}^{n}-\left(u_{i m}^{n-1}-u_{j m}^{n-1}\right)$, summing for $n=s, \ldots, m$ for arbitrary $s \in\{1,2, \ldots, m\}$, integrating by $\Omega$ and using the condition (2.7), we can obtain the following relation:

$$
\begin{aligned}
& E\left(u_{i m}^{m}-u_{j m}^{m}\right)+\frac{k}{2}\left(1-L_{1}\right) \sum_{n=s}^{m} \int_{\Omega}\left(\frac{u_{i m}^{n}-u_{j m}^{n}-\left(u_{i m}^{n-1}-u_{j m}^{n-1}\right)}{k}\right)^{2} d x+ \\
& +\sum_{n=s}^{m} \int_{\Omega}\left(\left|u_{i m}^{n}\right|^{p} u_{i m}^{n}-\left|u_{j m}^{n}\right|^{p} u_{j m}^{n}\right)\left(u_{i m}^{n}-u_{j m}^{n}-\left(u_{i m}^{n-1}-u_{j m}^{n-1}\right)\right) d x \leq \\
& \leq E\left(u_{i m}^{s-1}-u_{j m}^{s-1}\right),
\end{aligned}
$$

where

$$
\begin{gathered}
E\left(u_{i m}^{n}-u_{j m}^{n}\right)= \\
=\frac{1}{2} \int_{\Omega}\left|\Delta\left(u_{i m}^{n}-u_{j m}^{n}\right)\right|^{2} d x+\frac{1}{2} \int_{\Omega}\left(\frac{u_{i m}^{n}-u_{j m}^{n}-\left(u_{i m}^{n-1}-u_{j m}^{n-1}\right)}{k}\right)^{2} d x .
\end{gathered}
$$

It is evident that for arbitrary $\delta>0$ there exists $c_{2}(\delta)>1$ such, that

$$
|u-v|^{2} \leq \delta+c_{2}(\delta)|u-v|^{2}, \forall u, v \in R .
$$

We can obtain the following inequality from (3.4), when $s=1$ :

$$
\begin{aligned}
& k \sum_{n=1}^{m} \int_{\Omega}\left(\frac{u_{i m}^{n}-u_{j m}^{n}-\left(u_{i m}^{n-1}-u_{j m}^{n-1}\right)}{k}\right)^{2} d x \leq \delta \operatorname{Tmes} \Omega+C_{1} \frac{C_{2}(\boldsymbol{\delta})}{1-L_{1}}\|B\|_{V}^{2}+ \\
& +\frac{C_{2}(\delta)}{1-L_{1}} \sum_{n=1}^{m} \int_{\Omega}\left(\left|u_{j m}^{n}\right|^{p} u_{j m}^{n}-\left|u_{i m}^{n}\right|^{p} u_{i m}^{n}\right)\left(u_{i m}^{n}-u_{j m}^{n}-\left(u_{i m}^{n-1}-u_{j m}^{n-1}\right)\right) d x, \forall \delta>0 .
\end{aligned}
$$

By multiplying both sides of the equality (3.2) by $u_{i m}^{n}-u_{j m}^{n}$, summing for $n=1, \ldots, m$, integrating by $\Omega$ and using the condition (2.7), we have

$$
\begin{aligned}
& -k\left(1+\frac{L_{1}^{2}}{2 v}\right) \sum_{n=1}^{m} \int_{\Omega}\left(\frac{u_{i m}^{n}-u_{j m}^{n}-\left(u_{i m}^{n-1}-u_{j m}^{n-1}\right)}{k}\right)^{2} d x+ \\
& +\frac{k}{2} \int_{\Omega}\left(\frac{u_{i m}^{m}-u_{j m}^{m}-\left(u_{i m}^{m-1}-u_{j m}^{m-1}\right)}{k}\right)^{2} d x+ \\
& +\frac{k}{2} \int_{\Omega}\left(\frac{u_{i m}^{0}-u_{j m}^{0}-\left(u_{i m}^{-1}-u_{j m}^{-1}\right)}{k}\right)^{2} d x+ \\
& +\int_{\Omega} \frac{u_{i m}^{m}-u_{j m}^{m}-\left(u_{i m}^{m-1}-u_{j m}^{m-1}\right)}{k}\left(u_{i m}^{m}-u_{j m}^{m}\right) d x- \\
& -\int_{\Omega} \frac{u_{i m}^{0}-u_{j m}^{0}-\left(u_{i m}^{-1}-u_{j m}^{-1}\right)}{k}\left(u_{i m}^{0}-u_{j m}^{0}\right) d x+ \\
& +\int_{\Omega}\left(\frac{1}{2}\left(u_{i m}^{m}-u_{j m}^{m}\right)^{2}-\frac{1}{2}\left(u_{i m}^{0}-u_{j m}^{0}\right)^{2}\right) d x+ \\
& +k\left(1-\frac{v c_{\Omega}^{2}}{2}\right) \sum_{n=1}^{m} \int_{\Omega}\left|\Delta\left(u_{i m}^{n}-u_{j m}^{n}\right)\right|^{2} d x \leq 0,
\end{aligned}
$$


By (3.3) and due to the existence of a bounded absorbing set, we have

$$
E\left(u^{n}(T)\right) \leq c\left(\|B\|_{E}\right) \text { for } \forall T \geq 0,
$$

where $\|B\|_{E}=\sup _{v \in B}\|v\|_{E}$. Then from (3.6), we obtain the following

$$
\begin{aligned}
& k\left(1-\frac{v c_{\Omega}^{2}}{2}\right) \sum_{n=1}^{m}\left\|\Delta\left(u_{i m}^{n}-u_{j m}^{n}\right)\right\|^{2} \leq \\
& \leq C_{3}\left(\|B\|_{V}\right)+k\left(1+\frac{L_{1}^{2}}{2 v}\right) \sum_{n=1}^{m}\left\|\frac{u_{i m}^{n}-u_{j m}^{n}-\left(u_{i m}^{n-1}-u_{j m}^{n-1}\right)}{k}\right\|^{2} .
\end{aligned}
$$

Multiplying (3.5) by $v_{1}$ and summing it with (3.7), we have

$$
\begin{aligned}
& k \sum_{n=1}^{m} E\left(u_{i m}^{n}-u_{j m}^{n}\right) \leq \frac{v_{1} \delta T m e s \Omega}{2 v_{2}}+\frac{\tilde{C}\left(\|B\|_{V}, \delta\right)}{2 v_{2}}+ \\
& +k \frac{v_{1} C_{2}(\delta)}{2 v_{2}\left(1-L_{1}\right)} \sum_{n=1}^{m} \int_{\Omega}\left(\left|u_{j m}^{n}\right|^{p} u_{j m}^{n}-\left|u_{i m}^{n}\right|^{p} u_{i m}^{n}\right) \frac{u_{i m}^{n}-u_{j m}^{n}-\left(u_{i m}^{n-1}-u_{j m}^{n-1}\right)}{k} d x, \forall \delta>0,
\end{aligned}
$$

where

$$
v_{2}=\min \left\{v_{1}-1-\frac{L_{1}^{2}}{2 v}, 1-\frac{v c_{\Omega}^{2}}{2}\right\}
$$

and $v, v_{1}$ are chosen such that

$$
1-\frac{v c_{\Omega}^{2}}{2}>0, v_{1}-1-\frac{L_{2}^{2}}{2 v}>0 .
$$

Summing (3.4) for $s=1, \ldots, m$, using the condition $L_{1}<1$, (3.8) and lemma 2.2 from [15], we can obtain that

$$
\limsup _{i \rightarrow \infty} \limsup _{j \rightarrow \infty} E\left(u_{i m}^{m}-u_{j m}^{m}\right) \leq \frac{v_{1} m e s \Omega}{2 v_{2}} \delta+\frac{C\left(\|B\|_{V}, \delta\right)}{2 v_{2} T}, \forall \delta>0
$$

and consequently,

$$
\limsup _{i \rightarrow \infty} \sup _{q \in N} E\left(u_{(i+q) m}^{m}-u_{i m}^{m}\right) \leq \frac{2 v_{1} m e s \Omega}{v_{2}} \delta+\frac{2 C\left(\|B\|_{V}, \delta\right)}{v_{2} T}, \forall \delta>0, \forall T>0 .
$$

From the last relation we obtain, that for arbitrary $\varepsilon>0$ there exists $T=T(\varepsilon, B)$ and $\delta>0$ such that

$$
\limsup _{i \rightarrow \infty} \sup _{q \in N}\left[\frac{1}{2}\left\|\Delta\left(u_{(i+q) m}^{m}-u_{i m}^{m}\right)\right\|^{2}+\frac{1}{2}\left\|\frac{u_{(i+q) m}^{m}-u_{i m}^{m}-\left(u_{(i+q) m}^{m-1}-u_{i m}^{m-1}\right)}{k}\right\|^{2}\right] \leq \varepsilon,
$$

whence defining

$$
\tilde{u}_{m}(x, t)=u_{m}^{n}(x), \text { if }(\mathrm{n}-1) \mathrm{k}<\mathrm{t} \leq \mathrm{nk}, \mathrm{n}=1,2, \ldots, \mathrm{m} ; \text { a.e. in } \Omega
$$

and defining $\tilde{w}_{m}, \tilde{f}_{m}$ similarly, we obtain that

$$
\limsup _{i \rightarrow \infty} \sup _{q \in N}\left[\frac{1}{2}\left\|\Delta\left(\tilde{u}_{(i+q) m}-\tilde{u}_{i m}\right)(T)\right\|^{2}+\frac{1}{2}\left\|\left(u_{(i+q) m}-u_{i m}\right)_{t}(T)\right\|^{2}\right] \leq \varepsilon .
$$

Since (see, [9])

$u_{m} \rightarrow u$ weakly star in $H^{1}\left(0, T ; L^{2}(\Omega)\right) \cap L^{\infty}\left(0, T ; H_{0}^{1}(\Omega)\right)$,

$\tilde{u}_{m} \rightarrow u$ weakly star in $L^{\infty}\left(0, T ; H_{0}^{1}(\Omega)\right)$,

as $m \rightarrow \infty$, then passing to the limit as $m \rightarrow \infty$ in the inequality (3.9), we obtain (3.1).

Theorem 3.1 is proved.

Theorem 3.2. (the basic theorem) Assume that (2.4)-(2.12) hold. Then Problems (2.1)-(2.3) has a minimal global attractor which is invariant and compact.

The proof of Theorem 3.2. According to Theorem 2.7, under the conditions (2.4)-(2.12), problem (2.1)-(2.3) has a bounded absorbing set and by Theorem 3.1, the semigroup $\{S(t)\}_{t \geq 0}$, generated by this problem is asymptotically compact. Therefore according to Theorem 3.2 from [11], the problem (2.1)-(2.3) has a minimal global attractor, which is invariant and compact.

Theorem 3.2 is proved. 


\section{References}

[1] N. Kenmochi, A. Visintin, Asymptotic stability for nonlinear evolution problems with hysteresis, Europ. J. Appl. Math. 5 (1994), $39-56$.

[2] P. Krejci, Hysterezis and periodic solutions of semilinear and quasilinear wave equations, Math. Z. 193 (1986), $247-264$.

[3] P. Krejci, Asymptotic stability of periodic solutions to the wave equation with hysteresis, In: Models of hysteresis (A.Visintin, ed.), Longman, Harlow, 77-90, 1993.

[4] M. Hilpert, On uniqueness for evolution problems with hysteresis, Mathematical Models for Phase Change Problems, (J.F.Rodrigues,ed.), Birkhauser, Basel, 88, 1989, 377-388.

[5] A. Visintin, Hysteresis and Semigroups, in "Models of Hysteresis", A.Visintin, ed. Longman, Harlow, (1993) 192-206.

[6] A. Visinton, Quasilinear hyperbolic equations with hysteresis, Rend. Mat. Acc. Lincei, 15(9)(3-4) (2004), 235-247.

[7] A. Visintin, Differential Models of Hysteresis, Springer, 1993.

[8] J. L. Lions, Some Solution Methods for Nonlinear Boundary Problems, Moscow, Mir, 1972 (in Russian)

[9] S. E. Isayeva, The mixed problem for one semilinear hyperbolic equation with memory, Transactions of NAS of Azerbaijan XXX(1) (2010), 105-112

[10] R. Bouc, Modele mathematique d'hysteresis et application aux systemes a un degre de liberte, Thése, Marseille, 1966.

[11] O. A. Ladyzhenskaya, On the determination of minimal attractors for the Navier-Stokes equations and other partial differential equations, Uspekhi Mat. Nauk 42(6) (1987), 25-60. English translation: Russian Math. Surveys 42(6) (1987), 27-73.

[12] S.E. Isayeva, The existence of an absorbing set for one mixed problem with memory, Transactions of NAS of Azerbaijan XXXIII(1) (2013), 27-35.

[13] I. Chueshov, I. Lasiecka, Attractors for second-order evolution equations with a nonlinear damping, J. Dynam. Differential Equations 16(2) (2004), 469-512.

[14] I. Lasiecka, A. R. Ruzmaikina, Finite dimensionality and regularity of attractors for 2-D semilinear wave equation with nonlinear dissipation, J. Math. Anal. Appl. 270 (2002), 16-50.

[15] A. Kh. Khanmamedov, Global attractors for wave equations with nonlinear interior damping and critical exponents, J. Differential Equations 230 (2006), 702-719. 